\title{
Interpretation of experimental data near $\lambda$-transition point in liquid helium
}

\author{
J. Kaupužs * \\ Institute of Mathematics and Computer Science \\ University of Latvia \\ 29 Rainja Boulevard, LV-1459 Riga, Latvia
}

December 18, 2018

\begin{abstract}
The recently published experimental data for specific heat $C_{p}$ of liquid helium in zero gravity conditions very close to the $\lambda$-transition have been discussed. We have shown that these data allow different interpretations. They can be well interpreted within the perturbative RG approach and within our recently developed theory, as well. Allowing the logarithmic correction, the corresponding fits lie almost on top of each other over the whole range of the reduced temperatures $t$ (for bin averaged data) $6.3 \cdot 10^{-10}<t<$ $8.8 \cdot 10^{-3}$. However, the plot of the effective exponent $\alpha_{\text {eff }}(t)$ suggests that the behaviour of $C_{p}$, probably, changes very close to $T_{\lambda}$. To clarify this question, we need more accurate data for $t<10^{-7}$. In addition, we show that the experimental data for superfluid fraction of liquid helium close to $T_{\lambda}$ within $t \in\left[3 \cdot 10^{-7} ; 10^{-4}\right]$ can be better fit by our exponents $\nu=9 / 13 \simeq 0.6923$, $\Delta=5 / 13 \simeq 0.3846$ than by the RG exponents $\nu \simeq 0.6705$ and $\Delta \simeq 0.5$. The latter ones are preferable to fit the whole measured range $t \in\left[3 \cdot 10^{-7} ; 10^{-2}\right]$ where, however, remarkable systematic deviations appear. Our estimated value $0.694 \pm 0.017$ of the asymptotic exponent $\nu$ well agrees with the theoretical prediction $\nu=9 / 13$.
\end{abstract}

Keywords: liquid helium, $\lambda$-transition, critical exponents

\section{Introduction}

It is widely accepted to consider the measurements in liquid helium near $\lambda$-transition point $T=T_{\lambda}$ as a crucial test of validity of the theoretical predictions for the critical exponents, since these measurements are done with a high degree of accuracy much

${ }^{*}$ E-mail: kaupuzs@latnet.lv 
closer to the critical point than in any other experiments or numerical simulations. In particular, it is believed that accurate experimental measurements of specific heat $C_{p}$ of liquid helium very close to the $\lambda$-transition point in zero gravity conditions (in space) [1] provide a convincing evidence of overall correctness of the perturbative RG approach. The aim of our paper is to show that this conclusion is not unambiguous, since these experimental data as well as those of the superfluid fraction of liquid helium can be equally well or even better interpreted by a completely different set of critical exponents provided by our recently developed theory [2]

\section{Interpretation of the specific heat data}

It has been found in [1] that fits of experimental data for a wide range of reduced temperatures $5 \cdot 10^{-10} \leq t \leq 10^{-2}$ below $T_{\lambda}$ by using two slightly different ansatz,

$$
C_{p}=\frac{A^{-}}{\alpha} t^{-\alpha}\left(1+a_{c}^{-} t^{\Delta}+b_{c}^{-} t^{2 \Delta}\right)+B^{-}
$$

and

$$
C_{p}=\frac{A^{-}}{\alpha} t^{-\alpha}\left(1+a_{c}^{-} t^{\Delta}\right)+b_{c}^{-} t+B^{-},
$$

biased by the RG theoretical value of the correction-to-scaling exponent $\Delta=0.529$, provide well consistent values of the specific heat exponent $\alpha=-0.0127 \pm 0.0003$ in a good agreement with the value $-0.01294 \pm 0.0006$ of the variational perturbative theory [3] as well as in a worse, but still acceptable, agreement with more recent estimates $\alpha=-0.01126 \pm 0.0010$ [4] and $\alpha=-0.0146 \pm 0.0008$ [5]. Apart from the exponent $\alpha$, some other quantities have been determined and compared with the RG values in [1. However, the agreement is not so good to conclude that any theoretical approach, which does not agree with the perturbative $\mathrm{RG}$, is wrong. In particular, the experimental quantity $P=\left(1-A^{+} / A^{-}\right) / \alpha$ is $4.154 \pm 0.022$, whereas the recent $\mathrm{RG}$ calculation (Ref. 63 in [1]) yields $P=4.433 \pm 0.077$.

We have found that the measured data of $C_{p}$ [1] can be well reproduced also by an ansatz of the form

$$
C_{p}=t^{-\alpha}(C+A \ln t)\left(1+a t^{\Delta}\right)+B
$$

with fixed exponents $\alpha=-1 / 13$ and $\Delta=5 / 13$ proposed in [2, 6]. It is consistent with the idea that specific heat can have a logarithmic correction, as discussed in [2]. The power-like singularity is recovered at $A=0$. Note that in [2] (cf. Eq. (60) there) an ordinary term $\sim t^{-\alpha}$ is related the behaviour of the correlation function within the range of wave vectors $k \sim 1 / \xi$, where $\xi \sim t^{-\nu}$ is the correlation length, whereas the logarithmic term can appear due to the contribution of the region $k \gg 1 / \xi$. In this aspect, the ratio $A / C$ in (3) can be varied in a wide range of values.

From the raw data of [1] given in [7] we have produced the set of bin averaged data points by dividing each decade of the reduced temperature $t=1-T / T_{\lambda}$ in 10 segments of equal width when looking in the logarithmic scale. One binned 
data point has been obtained by an averaging over $C_{p}$ and $t$ values within one segment, and only the data for the smallest $t$ values within a twice wider interval $4.7 \cdot 10^{-10}<t<7.9 \cdot 10^{-10}$ have been merged together into one bin to reduce the statistical error. In our binning, the averaged data points come as close to $T_{\lambda}$ as $t \geq 6.3 \cdot 10^{-10}$, whereas those given in [7] extend only to $t \geq 7.94 \cdot 10^{-10}$.

The percent deviations from the least-squares fits to (3i) and (11) are shown in Fig. 1. The upper picture corresponds to (3) with fixed exponents $\alpha=-1 / 13$ and $\Delta=5 / 13$ and coefficients $C=-167.536, A=11.6593, a=0.19788$, and $B=$ 198.26, whereas the lower one represents the fit to (11) with exponents $\alpha=-0.01264$ (fit parameter) and $\Delta=0.529$ (fixed) and coefficients listed in Tab. II of [1]. As in some fits made in [1], we have assigned the error bars $0.02 \%$ to the bin averages which originally had smaller errors. In this way, we have reduced the impact of these data points, located at relatively large values of the reduced temperature $t$, where the asymptotic ansatz (3) is not very accurate. As we see from Fig. 1, both fits are almost identical in the whole range of the reduced temperatures. The fit with our exponents (top) is slightly worse at the largest $t$ values. It can be well understood, since (to ensure the stability of the fit parameters) we have neglected the subleading correction of the kind $t^{2 \Delta}$ included in the other ansatz (1). Besides, our fit is even slightly better at the smallest $t$ values: the mean percent deviation for 10 smallest $t$ values is $-0.425 \pm 0.690$ in our case of (3) and $-0.975 \pm 0.686$ in the case of (11). These deviations are reduced to $0.004 \pm 0.695$ and $-0.477 \pm 0.691$, respectively, when shifting the $T_{\lambda}-T$ values by $0.5 \mathrm{nK}$ within the experimental error bars [1].

Note that only the possibility and not the necessity of the logarithmic correction follows from the theory [2]. However, the presence of the logarithmic correction for specific heat, perhaps, is a quite general feature: the logarithmic singularity (as a special case of the logarithmic correction when $\alpha=0$ ) of specific heat is a rigorously stated fact in 2D Ising model [8], and our Monte Carlo simulation data for 3D Ising model [6] also supports the logarithmic singularity. Our analysis of the experimental data for the superfluid fraction, made in Sec. 3, gives one more argument: it suggests that the exponent $\nu$ is remarkably larger than 0.6705. According to the known scaling relation $\alpha+d \nu=2$ [8], the exponent $\alpha$ then should be remarkably more negative than -0.0115 . It well coincides with the measured data [1] in the whole range of the reduced temperatures only if the pure power is perturbed by a logarithmic correction, as proposed by ansatz (3).

Considering $\alpha$ as a fit parameter in (3), we obtain a value $\alpha=-0.0848 \pm 0.0039$, which is quite close to our theoretical prediction $\alpha=-1 / 13 \simeq-0.0769$. The small systematic deviation could be caused by the error of the asymptotic ansatz (3) at the largest $t$ values. This problem cannot be reliably solved by adding more correction terms or by narrowing the range of the fit, since the minimum of the $\chi^{2}$ for such fits is very shallow, i. e., the results become poorly defined.

Alternatively, we have fit the data within a moving window $t \in\left[t_{i} ; 100 t_{i}\right]$ of the reduced temperatures to the simplest possible ansatz

$$
C_{p}=A t^{-\alpha}+B
$$



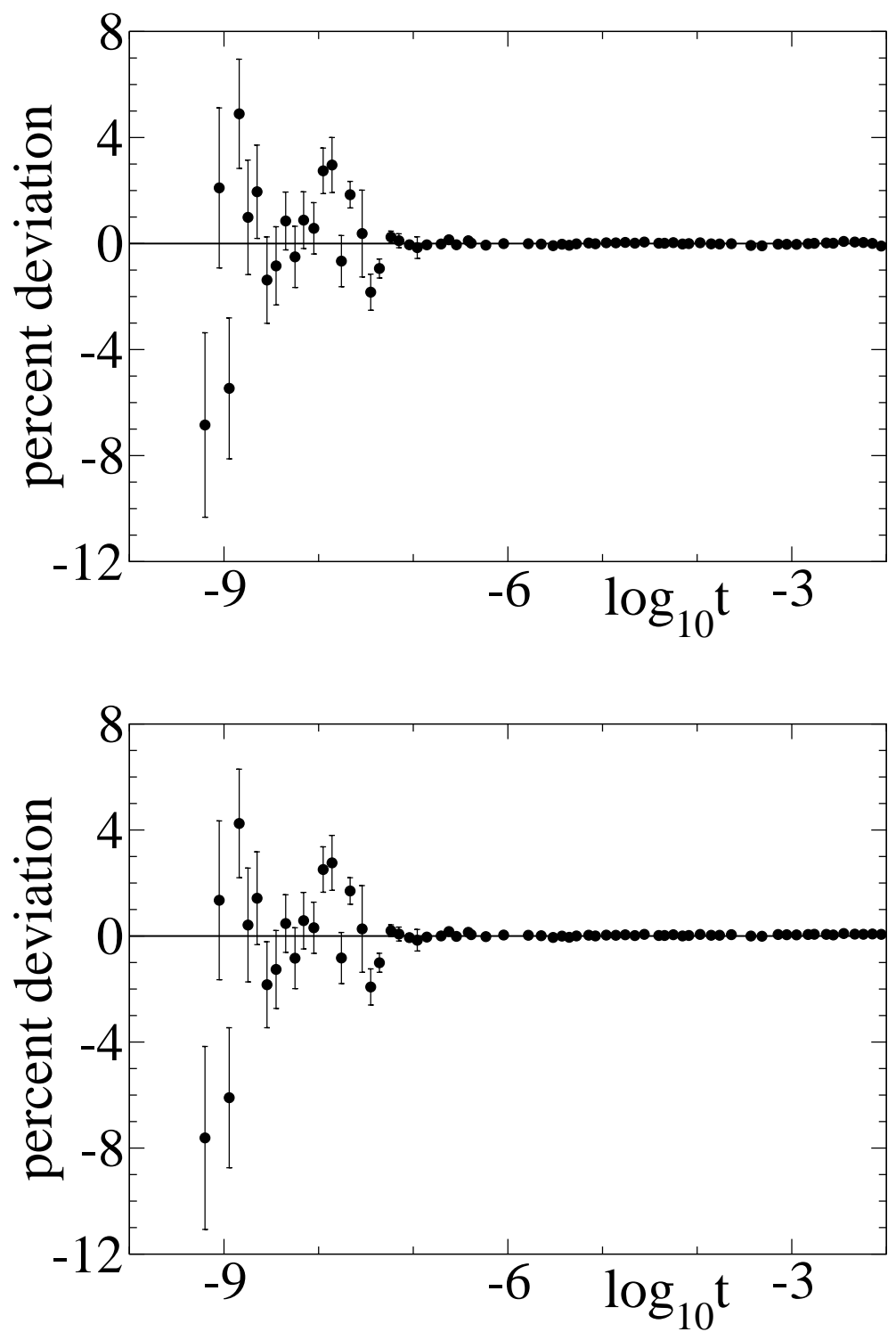

Figure 1: Percent deviation of the fitted $C_{p}$ data points from the ansatz (3) with fixed exponents $\alpha=-1 / 13$ and $\Delta=5 / 13$ (top) and from the ansatz (10) with the fit parameters found in [1] (bottom). 

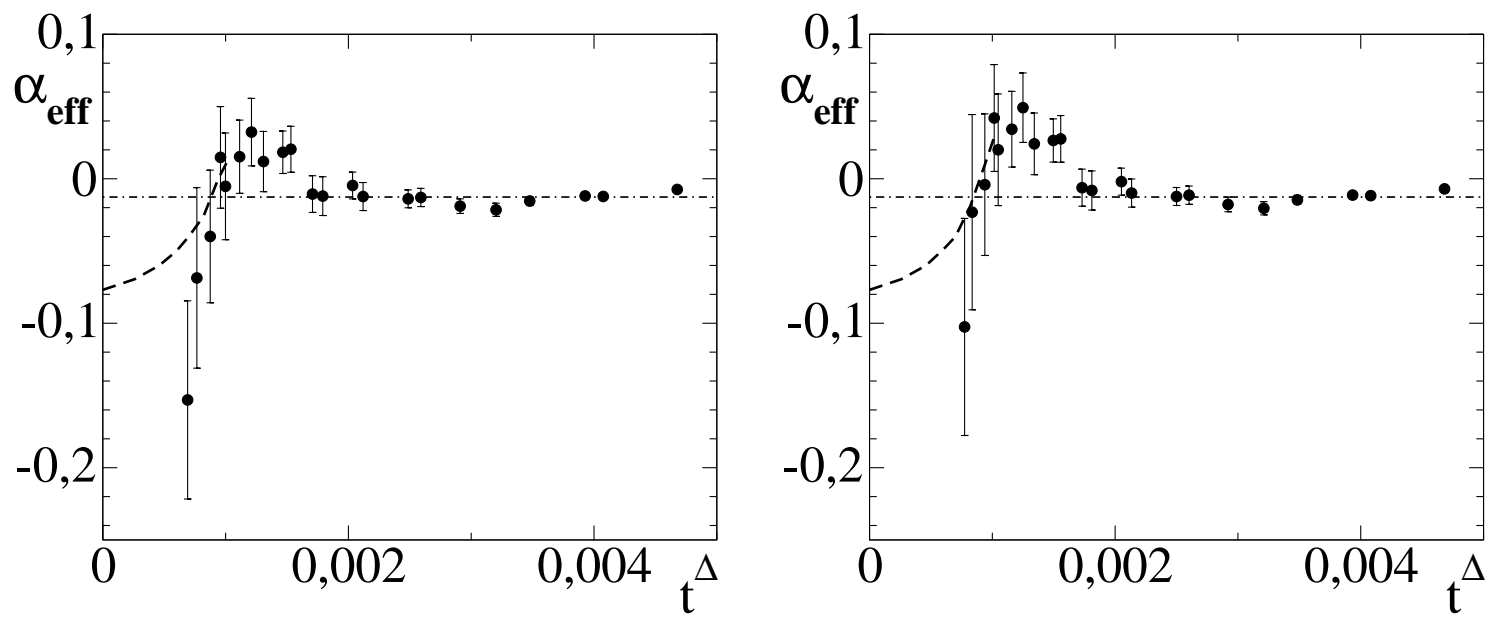

Figure 2: The effective exponent $\alpha_{\text {eff }}$ vs $t^{\Delta}$ estimated by fitting the bin averaged data within $\left[t_{i} ; 100 t_{i}\right]$ to the ansatz (44) with unbiased (left) and shifted according to $T_{\lambda} \rightarrow T_{\lambda}+0.5 \mathrm{nK}$ (right) values of the reduced temperatures. The horizontal dot-dashed line indicates the $\alpha$ value -0.01264 found in [1, whereas the dashed curve shows schematically a behaviour expected from [2].

where $t_{i}$ corresponds to the $i$-th bin averaged data point. It yields the effective exponent $\alpha_{\text {eff }}(t)$, where $t$ belongs to the considered interval. For convenience, we have defined it as $t=\sqrt{t_{\min } t_{\max }}$, where $t_{\min }=t_{i}$ is the minimal and $t_{\max }$ is the maximal $t$ value in the interval. By this method, the result converges to the true value of $\alpha$ at $t \rightarrow 0$ irrespective to the error of the asymptotic ansatz at finite $t$. It works also when the logarithmic correction is present, only in this case the convergence is very slow, like $\alpha-\alpha_{\text {eff }}(t) \sim 1 / \ln t$ at $t \rightarrow 0$. The only problem is that this method requires that the measurement errors both for $C_{p}$ and $t$ remain sufficiently small when approaching $T_{\lambda}$.

We have plotted in Fig. 2 the results for $\alpha_{\text {eff }}$ depending on $t^{\Delta}$ (with our exponent $\Delta=5 / 13$ ) obtained by using the unbiased values of $t_{i}$ (left), as well as the shifted values $t_{i}^{\prime}=t_{i}+2.3 \cdot 10^{-10}$ (for the same sets of measured points) corresponding to $T_{\lambda} \rightarrow T_{\lambda}+0.5 \mathrm{nK}$ (right). In spite of the large error bars, these plots show certain trend, where the effective exponent $\alpha_{\text {eff }}(t)$ tends to decrease below the (RG) value -0.01264 found in [1]. Moreover, we have verified that the same trend is observed in both cases when only the odd and only the even raw measurements (the original values listed in [7]) are used. Hence, we cannot exclude any striking scenario. For instance, $\alpha_{\text {eff }}(t)$ could converge to our asymptotic value $\alpha=-1 / 13$, as indicated by dashed lines, particularly, if we allow a small shift in $T_{\lambda}-T$ values (right picture) within the experimental error bars. However, such a behaviour would mean that the logarithmic correction is absent, since the convergence is rather fast. It would imply also that the estimation of $\alpha$ from the fit over the whole measured range is not valid: formally, such a fit looks good, but it effectively ignores the systematic deviations at the smallest $t$ values where the error bars are larger. 
Due to the experimental errors, the results of our analysis of the effective exponent are not conclusive, they only point to a possible scenario. From an intuitive point of view, it does not seem plausible that such a remarkable change in the behaviour of the system could take place at so small reduced temteratures $\left(t<10^{-7}\right)$. However, since the deviations from the asymptotic scaling law are caused by the corrections to scaling, an essential parameter is $t^{\Delta}$ rather than $t$, and the values of $t^{\Delta}$ in Fig. 2] are not so extremely small. Besides, the critical region, where an asymptotic ansatz is valid, can be as narrow as $t^{\Delta} \sim 0.001$ even in a simple mean field model. An example is given in [9] (p. 75). From this point of view, it is possible that the deviations in Fig. 2 represent a real physical effect and not an artifact. On the other hand, random deviations in Fig. 1 too often are as large as 2 standard deviations or even larger, therefore the unusual behaviour of $\alpha_{\text {eff }}$ in Fig. 2 can be ascribed also as an artifact.

\section{Interpretation of the experimental data for the superfluid fraction}

Here we discuss the experimental data for the superfluid fraction $\rho$ in liquid $H e$. It decreases asymptotically (at $t \rightarrow 0$ ) as $\rho \sim t^{\zeta}$. It is believed (see [10] and references therein) that the exponent $\zeta$ is equal to the correlation length exponent $\nu$ for the $3 \mathrm{D}$ $X Y$ model. In [2], the superfluid fraction of ${ }^{4} \mathrm{He}$ measured in [10] has been discussed with an aim to compare the experimentally observed behaviour at the temperatures closest to $T_{\lambda}$ with our theoretical prediction $\nu=9 / 13$ [2].

The data listed in [11] allow a more precise comparison. For this purpose, first we have fit these data to the asymptotic ansatz

$$
\rho=A t^{\nu}\left(1+a_{1} t^{\Delta}+a_{2} t^{2 \Delta}\right)
$$

including two corrections to scaling. Similar fits over the whole measured range $t \in\left[3 \cdot 10^{-7} ; 10^{-2}\right]$ have been considered in [10, 11]. Note that at $\Delta=0.5$, used in [10, 11], the second order correction reduces to the analytical one, and (15) differs from the ansatz of [10, 11] only by a remainder term of higher order. The overall fits discussed in [10, 11] yield $\nu \simeq 0.6705$ in agreement with the RG prediction and in disagreement with our value $\nu=9 / 13$. However, these fits look really good only within $t \in\left[10^{-5} ; 10^{-2}\right]$, whereas remarkable systematic deviations appear at smaller $t$ values. This phenomenon was discussed in [11] and no reasonable explanation was found. In particular, the effect of gravity is negligible in these experiments [1] and the $\pm 20 \mathrm{nK}$ uncertainty in $T_{\lambda}-T$ also does not explain these systematic deviations.

Our theory 2 provides an explanation. First, the data cannot be well fit within the whole measured range $t \in\left[3 \cdot 10^{-7} ; 10^{-2}\right]$ simply because the critical region, where the asymptotic expansion in $t$ powers is valid, is much narrower than $10^{-2}$. Second, the data can be well fit to (5) with our exponents $\nu=9 / 13$ and $\Delta=5 / 13$ within a reduced range $t \in\left[3 \cdot 10^{-7} ; 10^{-4}\right]$, which means that the measured data for $t<10^{-5}$ are not anomalous, but the region of validity of (5) is as narrow as $10^{-4}$. 

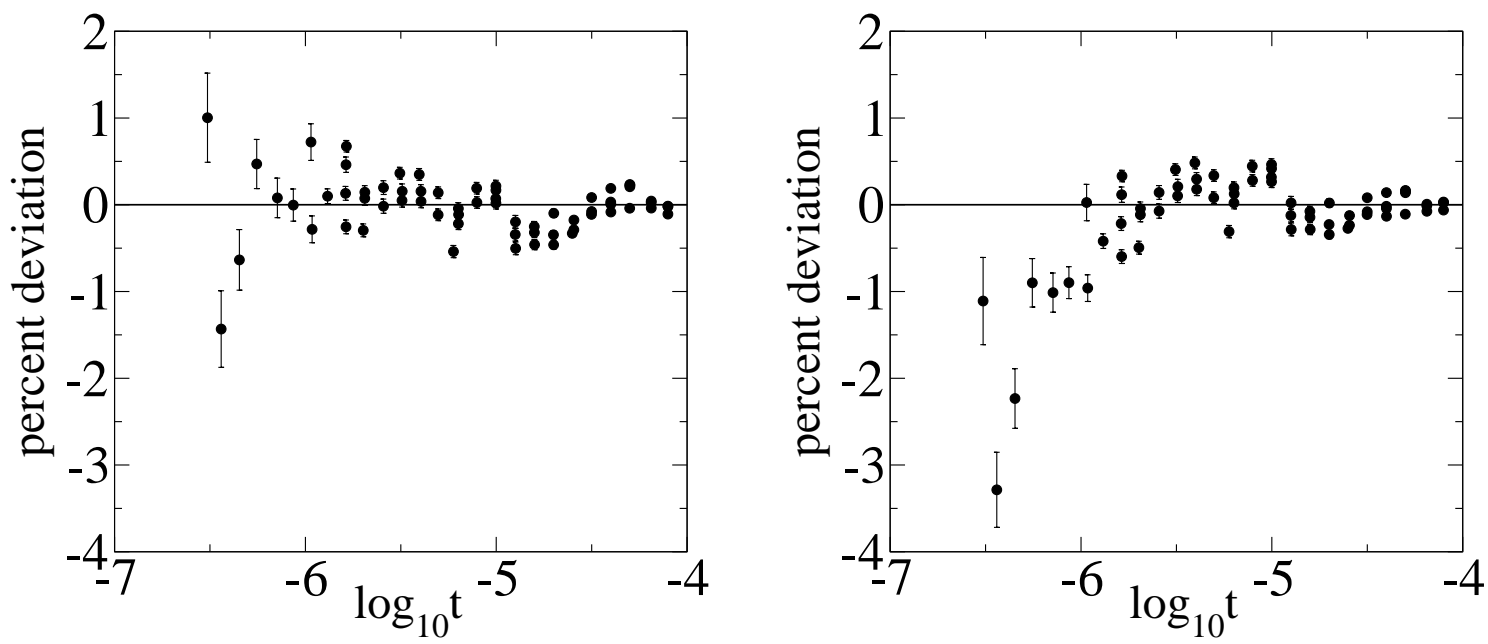

Figure 3: Percent deviation of the experimental $\rho$ (superfluid fraction) data points from the least squares fit to ansatz (5) within $t \in\left[3 \cdot 10^{-7} ; 10^{-4}\right]$ with our exponents $\nu=9 / 13, \Delta=5 / 13$ (left) and with the RG exponents $\nu=0.6705, \Delta=0.5$ (right).

The percent deviations from the least-squares fits within $t \in\left[3 \cdot 10^{-7} ; 10^{-4}\right]$ with our (left) and RG (right) exponents are shown in Fig. 3. As we see, in our case there are no essential systematic deviations, whereas in the RG case they are observed like in the case of the fit over the whole measured range [10, 11.

Similarly as in Sec. 2, we have evaluated also the effective exponent $\nu_{\text {eff }}(t)$ as the local slope of the $\ln \rho$ vs $\ln t$ plot within $\left[t_{i} ; 5 t_{i}\right]$, where $t_{i}$ is the reduced temperature of the $i$-th measurement and $t$ is the middle point of the fitted interval in the logarithmic scale. The results depending on $t^{\Delta}$ (with our value $\Delta=5 / 13$ ) are shown in Fig. 4. Evidently, the effective exponent tends to deviate above the value 0.6705 (dot-dot-dashed line) obtained in [10, 11. On the other hand, the fit of this plot to a parabola (solid line) gives the asymptotic estimate $\nu=0.694 \pm 0.004$ in excellent agreement with our theoretical value $9 / 13 \simeq 0.6923$. However, taking into account the $\pm 20 \mathrm{nK}$ uncertainty in the $T_{\lambda}$ value [10], the error bars become larger, i. e., $\nu=0.694 \pm 0.017$.

\section{Conclusions}

Although the opinion dominates in publications that the perturbative RG theory is strongly confirmed by very accurate measurements of the specific heat and the superfluid fraction in liquid helium near the $\lambda$-transition point, our current analysis shows that these experimental data can be well understood and interpreted also within our recently developed theory [2].

In summary we conclude the following:

1. The critical exponents of the perturbative RG theory look preferable from a 


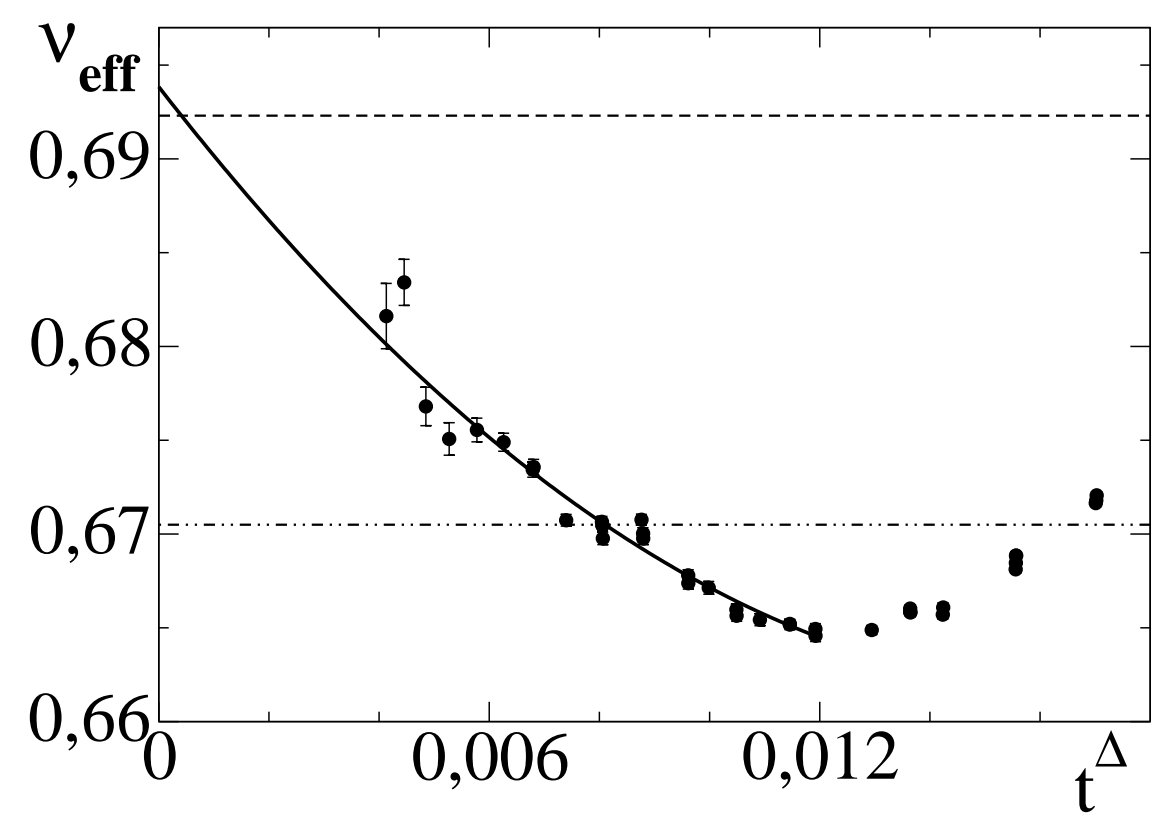

Figure 4: The effective exponent $\nu_{\text {eff }}$ vs $t^{\Delta}$ determined from the local slopes of the $\ln \rho$ vs $\ln t$ plot. The lower dot-dot-dashed line indicates the (RG) value 0.6705 obtained in [10, 11, whereas the upper dashe line shows our theoretical value $\nu=9 / 13$. The solid curve represents the least-squares fit to a parabola.

point of view that all measured data points must be necessarily fit on one curve and no logarithmic corrections are normally expected. However, if we allow the logarithmic correction to specific heat $C_{p}$, our theory also provides a good fit of $C_{p}$ data for the whole measured range. Our fit then is slightly worse at the largest reduced temperatures $t \sim 10^{-2}$ and better at the smallest ones $t \sim 10^{-9}$. As discussed in Sec. 2, the existence of the logarithmic correction is partly supported by our estimation of the exponent $\nu$ (Sec. 3), as well as by some general argument.

2. The analysis of the effective exponent $\alpha_{\text {eff }}$ indicates that the behaviour of $C_{p}$ could be remarkably changed very close to $T_{\lambda}$, in such a way that the true asymptotic singularity is power-like (without the logarithmic correction) with the exponent which more probably is closer to our value $\alpha=-1 / 13$ than to the $(\mathrm{RG})$ value -0.01264 obtained in [1]. However, this effect can be ascribed also as an artifact caused by the measurement errors. Further improvement of the experimental accuracy for $t<10^{-7}$ would be very helpful to clarify this question.

3. As compared to the RG exponents, our critical exponents are better consistent with the closest to $T_{\lambda}$ data $\left(t \in\left[3 \cdot 10^{-7} ; 10^{-4}\right]\right)$ for the superfluid fraction (cf. Figs. [3] and 44). A self consistent estimation in this case yields $\nu=0.694 \pm$ 0.017 in agreement with our theoretical prediction $\nu=9 / 13 \simeq 0.6923$. Since 
the fit over the whole measured range $\left(t \in\left[3 \cdot 10^{-7} ; 10^{-2}\right]\right)$ in no case is really good, we argue that our way of estimation is preferable.

\section{Acknowledgments}

This work partly has been done during my stay in 2005 at the Institute of Physics of Rostock University, Germany.

\section{References}

[1] J. A. Lipa, J. A. Nissen, D. A. Stricker, D. R. Swanson, T. C. P. Chui, Phys. Rev. B 68, 174518 (2003)

[2] J. Kaupužs, Ann. Phys. (Leipzig) 10, 299 (2001)

[3] H. Kleinert, Phys. Rev. D 60, 085001 (1999)

[4] H. Kleinert, Phys. Lett. A 277, 205 (2000)

[5] M. Campostrini, M. Hasenbusch, A. Pelissetto, P. Rossi, E. Vicari, Phys. Rev. B 63, 214503 (2001)

[6] J. Kaupužs, Proceedings of SPIE 5471, 480 (2004); see also e-print cond-mat/0405197

[7] EPAPS Document No. E-PRBMD0-68-041341, see EPAPS homepage http://www.aip.org/pubservs/epaps.html

[8] Rodney J. Baxter, Exactly Solved Models in Statistical Mechanics, (Academic Press, London, 1989)

[9] R. Mahnke, J. Kaupužs, I. Lubashevsky, Physics Reports 408, 1 (2005)

[10] L. S. Goldner, G. Ahlers, Phys. Rev. B 45, 13129 (1992)

[11] L. S. Goldner, N. Mulders, G. Ahlers, Journal of Low Temperature Physics 93, 131 (1993) 